\title{
EL SINDICATO EN ITALIA: ¿UNA CRISIS DE REPRESENTACIÓN?
}

\author{
Paolo Giovannini
}

\section{INTRODUCCION}

En la actualidad, y en este final de los afios ochenta, quizá sea posible establecer un primer balance acerca de un acontecimiento crucial de nuestros tiempos: el de la crisis del sindicato en un país que, como Italia, siempre se ha caracterizado por una presencia fuerte y amplia de las organizaciones de representación del movimiento obrero. En estos pocos años, ¿qué pudo ocurrit que fuera tan radical como para transformar a un protagonista indiscutido de la escena econónica y política itajiana en un actor que desempeña unos papeles cada vez más marginales? Realmente, than cambiado tan profundamente la composición social y la organización productiva de la Italia contemporánea como para socavar esta institución, o estamos ante todo frente a un proceso de mutación cultural e ideológica, que ha debilitado su garra y empañado su imagen? Ciertamente, la crisis ha sido y es lo bastante profunda y extensa como para que, incluso en ambientes fuera de toda sospecha, hasta se haya llegado a interrogarse sobre un posible declive histórico del síndicato $o$, al menos, acerca de una redefinición marginal de su papel, bastante alejado de la presencia y potencia que le conocimos en años todavía cercanos. ${ }^{1}$

En este artículo intentaré afrontar el problema de la crisis sindical considerando los aspectos que me parecen de mayor relieve y que se relacionan

1. Véase, a título de ejempio, el debate organizato por la revista oficial de la CGIL sobre el tema: ¿Declive del sindicato? («Rasegna sindacale», otoño-invierno 1984-1985). Para unas evaluaciones más optimistas, véase también MaNGH, 1987. 
directamente con el tema de la representación. En primer lugat, se trata de intertogarse acerca de las transformaciones cuantitativas y cualitativas que se han producido en la base social de referencia del sindicato, acerca de cómo y cuánto éstos inciden sobre lo que, por parte sindical, se quiere; se pretende, se presume tepresentar; en segundo lugar, de evaluar cómo y a través de qué canales se expresan exigencias e intereses, y en qué medida los procesos de decisión maduran adecuadanente y en qué nivel lo hacen. Este segundo aspecto, estrechamente ligado al tema de la democracia interna del sindicato, será abordado, por razones de espacio, solamente cuando sea necesario para aclarar algunos puntos del primero, con la convicción, sin embargo, de que reflexionar sobre la representación también significa, inevitablemente, plantearse el problema de volver a pensar sobre la organización (Romagnoli, 1981).

Como veremos, sin embargo, resulta más difícil separar los aspectos cualitativos de los cuantitativos en la crisis del sindicato, es decir, reflejar separadamente su dificultad de representación y su caída de representatividad, según la distinción clásica en los estudios sobre la democracia (Cella, 1981; Sartori, 1987). Bien es cierto que los años ochenta han proporcionado una cantidad superabundante de traterial de teflexión sobre ambos aspectos, tal como lo demuestra la amplia literatura disponible acerca del tema y como lo demuestran bastante mejor aún las nuevas representaciones sindicales salidas a la luz en estos últimos tiempos y conocidas genéricamente bajo el nombre de Cobas (Comités de base). ${ }^{2}$ A este sindicalismo extraconfederal no se le podrá hacer sino una breve mención, no sólo porque la atención prevalente debe concederse a la crisis de representación del sindicalismo confederal, sino también porque, como lo han sostenido justamente Carrieri y Donolo (1989), aquí nos encontramos en presencia de unos procesos todavía en fase de consolidación y de unos fenómenos de organización que guardan una mayor relación con la lógica del conflicto de intereses, y de intereses profesionalmente definidos, que con la cuestión de la representación social.

\section{LAS TRANSFORMACIONES SOCIALES}

La segunda mitad de los años setenta representa, a mi parecer, un paso importante en el proceso de trutación que caracteriza la sociedad, la política

2. La más reciente discusión sobre este tema es la que tuvo lugar en Siena el 20 y 21 de enero de 1989, Después de los Cobas: cuestiones sobre la representatividad sindical. 
y la economía italianas. Las elecciones de 1975 y las de 1976 , con un fuerte avance del PCI incluso en las áreas urbanas y modernas del país, representan el último fruto político del éxito y de la relativa begemonía cultural de un modelo productivo fundado sobre el predominio del sector industrial y de la gran emptesa, y de un modelo social centrado en la clase obrera y en sus organizaciones de representación (el sindicato confederal y el partido comunista). A partir de entonces, primero de forma soterrada y después bajo formas y modos cada vez más patentes, van energiendo en Italia los petfiles de un nuevo orden productivo y de una nueva configuración social. El sector terciario, que ya en el censo de 1971 empezaba a amenazar la primacía apenas conquistada en 1961 por la industria, sigue su irresistible ascensión, cambiando tápidamente la faz ocupacional y social de la Italia de los años ochenta. ${ }^{3}$ Crecen, en paralelo, las capas sociales ligadas al sector terciario, tanto en su componente público o parapúblico o en los sectores tradicionales de intermediación comercial, como en aquellos nuevos fomentados por el desarrollo industrial (el llamado terciario avanzado). ${ }^{4}$ Sin embargo, las transformaciones en el secundario están a punto de modificar radicalmente los equilibrios sociales y políticos alcanzados entre finales de los años sesenta y primetos del setenta. Por una parte, se asiste a un progresivo declive de la gran industria, con una constante pérdida de personal, a partir de 1974, en las empresas mayores de 500 empleados. El bloqueo de hecho del turnover y la política de descentralización productiva puesta en marcha a partir de finales de los años sesenta conducen a una sustancial refundición de la organización productiva incluso en las áreas de mayor concentración industrial del noroeste del país, donde crece y se difunde capilarmente en el territorio una red de pequeñas y medianas empresas, y también de centros de servicios para las empresas (Varaldo, 1979; Salvati, 1984). Las transformaciones de mayor relieve, según muestro punto de vista, tienen lugat, sin embargo, en las áreas de desarrollo difuso del centro y nordeste. En ellas se había afanzado, casi desde la inmediata posızuerra, un modelo productivo

3. Distribución porcentual de los ocupados por sectores de actividad.

\begin{tabular}{llllll}
\hline Sector & 1951 & 1961 & 1971 & 1981 & 1986 \\
\hline Primatio & 42.2 & 29.0 & 17.2 & 13.3 & 10.7 \\
Secundario & 32.1 & 40.0 & 44.3 & 37.2 & 32.8 \\
Terciario & 25.7 & 30.6 & 38.5 & 49.5 & 56.5 \\
\hline
\end{tabular}

Para un cuadro actualizado de la ocupación y dal mercado del trabajo en los años ochenta, cf. ISTAT-AIS, 1988.

4. Puede encontrarse un análisis de conjunto de tas transformaciones en la estratificación social en: Sylos Labint, 1986; Ascoli-Catanzato, 1987. 
fundado en la pequeña y muy pequeña empresa, que había crecido y se había difundido de forma monocultural y sobre una base territorial. Mientras en las áreas de más antiguo asentamiento industrial el proceso productivo venía organizándose en torno a la gran empresa — según el clásico modelo inglés de expansión del capital--, en las zonas centro y notdeste del país la industrialización seguía una vía alternativa a la de producción de masa (Bagnasco, 1977; Sabel-Zeitlin, 1982), la de la especialización flexible, promoviendo, y al mismo tiempo usufructuando, una movilización económica y social desde abajo, que encontraba como principales protagonistas a las clases campesinas (aparceros y pequeños cultivadores) «liberadas» del campo, pero también a las capas artesanales y comerciales de las pequeñas y medianas ciudades de antigua tradición comunal (Bagnasco, 1988). Si bien, hasta los primetos años del setenta, el desarrollo de aquellos distritos industriales» (Becattini, 1987) había terido lugar a la sombra de la gran empresa y era considerado a menudo y apresuradamente como una de las muchas caras de la descentralización productiva, en la segunda mitad de los años setenta, y especialmente en los actuales ochenta, para la mayoría ya parece claro que estamos frente a un fenómeno nada transitorio, sino de naturaleza estructural. Esta otra vía diferente hacia el desarrolto del capital, con su innegable éxito, termina por modificar profundamente la composición social de la clase obrera de la industria (ya veremos sus consecuencias sobre el sindicato) y, sobre todo, lleva a una nueva afirmación sobre el plano ideoIógico y cultural de los valores competitivos y de mercado típicos del modelo de desarrollo difuso (Giovannini, 1985). Entra en crisis una de las opciones políticas e ideológicas que habían caracterizado a la sociedad italiana durante una larga fase, es decir, aquella opción igualitaria y solidaria que se había expresado tan fuertemente en el éxito del sindicalismo «de clase» a partir de finales de los años sesenta. Nuevos valores, fundamentalmente antisolidarios y antiigualitarios, recorten tápidamente la sociedad italiana y parecen conquistarla (Antoniazzi, 1986). Se abre una fase, inconclusa todavía, de intensa dinámica económica y social, que involucra estratos antiguos y nuevos. Las capas y áreas más débiles del país parecen aisladas y descuidadas, mientras que los sujetos sociales y los grupos profesionales de mayor capacidad contractual (de hecho o de derecho) buscan espacio y reconocimiento económico por vías individuales o mediante el recurso a nuevas formas de tutela de los intereses (Cobas, Gildas, y en general el sindicalismo autónomo). 
El sindicato en Italia: ¿una crisis de representación?

\section{EFECTOS SOBRE EL SINDICATO}

Si estas transformaciones, apenas esbozadas, son las principales sufridas por la sociedad italiana en estos últimos 10 o 15 años, ¿qué reffejos, tanto directos como indirectos, habrán tenido sobre la representación del síndicalismo confederal? Una primera evaluación muy general muestra cómo tales procesos de transformación vuelven a cliseñar la estratificación social en un sentido no favorable a la membership sindical, al menos en sus connotaciones tradicionales (Paci, 1982). De hecho, en estos años han ido creciendo los componentes sociales en los que el sindicato era débil, ape. nas presente y con dificultades de afiliación no fácilmente superables; y, mientras tanto, han disminuido los que alimentaban masivamente su base y garantizaban su capacidad conflictual. La expansión del sector terciatio, es* pecialmente el público, de hecho ha significado sociológicamente un crecimiento de los componentes sociales de clase media y de empleados, fun* damentalmente ajenos a la lógica del sindicalismo, sobre todo en su versión «de clase». En el sector privado, ésta ha producido un aumento del númeto de los trabajadores autónomos, probablemente provenientes del trabajo asalariado, al menos en las zonas más desarrolladas del centro y norte de Italia: tal como to han demostrado numerosas investigaciones, en cierto punto de la evolución del ciclo vital (personal, familiar y profesional), no es raro, de hecho, el paso (o la fuga) de la condición de trabajador dependiente a la de independiente, no sólo en los servicios sino también en las industrias (artesanado industrial, microempresas, etc.) (Paci, 1973; Bagnasco, 1988). Nos encontramos en presencia de un fenómeno ampliamente imprevisto no sólo por el análisis económico y sociológico tradicional, sino sobre todo por los pensadores influidos por el marxismo 0 , de alguna forma, cercanos al movimiento obrero, pues siempre han razonado en términos de proletarización creciente (es decir, de crecimiento progresivo del trabajo subordinado), sin tener en cuenta que en los países más desarrollados podría llegar a concentrarse, mediante una división internacional del trabajo, una cuota inusualmente alta de trabajo autónomo o microempresatial, en paralelo con unos procesos de descentralización de las producciones industriales de masa hacia los países subdesartollados.

Sin embargo, quizá sea lo que está ocurriendo en el sector del trabajo

5. En el sector público las organizaciones sindicales más fuertes siempre han sido las de la CSIL, UIL y los sindicatos autónomos. En. los últimos años, la situación se ha vuelto más equilibrada con un buen mantenimierto de la CGIL y un fuerte crecimiento de la UIL: cf. tabla 1, y también Frrraresi, 1981; Cella-Treu, 1989. 
industrial lo que vaya a tener los efectos de mayor relieve sobre la dinámica de las afiliaciones al sindicato. En primer lugar, cambia la telación entre los componentes obreros y administrativos dentro de la ocupación industrial, ya que se extienden la automación y la informatización del trabajo obrero; en las empresas, el eje central se ha desplazado de las funciones directamente productivas hacia las proyectivas, de gestión y comercialización; y finalmente, el trabajo obrero viene siendo descentralizado sistemáticamente a nivel internacional, en busca de menores costes y niveles más bajos de control sindical. En la mediana o gzan empresa, por tanto, asistimos a un doble movimiento negativo: reducción del empleo dependiente en su conjunto y, dentro de éste, reducción más que proporcional de su componente obrero. Para el sindicato, esto supone una retracción de su base potencial más natural (la obrera) y una extensión relativa del componente administrativo y técnico, nunca realmente afecto al consenso sindical más allá de algunos momentos, excepcionales, de fascinación y, por el contrario, recorrido a menudo por veleidades de autonomía, cuando no de corporativismo, que algunas veces han dado lugar a verdaderas acciones de secesión sindical. ${ }^{\circ}$

Lo que, por el contrario, no parece estar en crisis, al menos hasta los tiempos más recientes, es el mundo variopinto de la pequeña empresa. Su clase obrera, fraccionada entre miles y miles de empresas peto presente de forma más masiva en algunas áreas del país (la llamada Tercera Italia), no sólo no disminuye en cantidad, sino que ni siquiera manifiesta síntomas de desafección en las confrontaciones del sindicato. Si bien es verdad que, a nivel de empresa, las dificultades de afiliación, de representación y de lucha sindical permanecen bastante fuertes (tal cono siempre ba ocurrido en las unidades con pocos asalariados, por otra parte), en su conjunto tanto la cuota de sindicalización entre los obretos de la pequeña empresa como su expresión conflictiva no parecen experimentar una crisis grave. Es que, en aquellas zonas de desarrollo difuso, en aquellos «distritos industriales», las relaciones entre empresas y trabajadores se crean y se viven más en un plano territorial que en el plano empresarial, como bien lo ha demostrado Trigilia (1985). Tales zonas, caracterizadas más que otras por la presencia de unas subculturas políticas enraizadas, sean comunistas o católicas, conservan una práctica de afiliación a los respectivos sindicatos (CGIL y CISL) que, ciertamente, se halla relacionada con las identidades políticas paralelas, pero que se explica también en términos directamente sindicales. Nos hallamos ante una tepresentación capaz de expresarse eficazmente en el territorio

6. Es emblemática al respecto la famosa «marcha de los 40.000 (o de los «cuadros») en Turín, en 1980. 
y que da vida a unos sistemas de negociación territorial satisfactorios, donde se encuentran normalmente envueltos, en conjunto con los sindicatos y las asociaciones industriales, los entes locales en una especie de neocorporativismo de base local. Por tanto, si bien en general las dificultades del sindicato encuentran su explicación en una caída cuantitativa de la clase obrera de la industria, deben verse diferentemente los trends de algunas áreas del país (más directamente ligadas a los destinos de ta gran empresa) y los de otras zonas en las que una clase obrera de pequeĩa empresa conserva todavía intacta, por ahora, su presencia y mantiene una telación de consenso con el sindicato, en tanto en cuanto es el protector genérico pero eficaz de las condiciones de vida en la zona antes que de las condiciones de trabajo en la propia empresa.

Desde el punto de vista obrero y sindical, la difusión de modalidades productivas fundadas en la pequeña empresa no presenta, por tanto, grandes efectos negativos: esto no significa, sin embargo, que este desplazamiento del baricentro económico ( $\mathrm{y}$ también político y cultural) desde la grande hacia la pequeña dimensión haya sido y sea sin consecuencias para el actor sindical. Intentaremos argumentar un poco esta afrmación. Ya se sabe que los sectores que alimentan esta modalidad de desarrollo son, esencialmente, los Ilamados sectores «típicos", expuestos a la competencia internacional y a menudo sujetos a las cambiantes leyes de la moda. La «especialización flexible» es la condición de base para la supervivencia en el mercado (Piore-Sabel, 1985). Culturalmente, este cuadro alimenta y "premia» los valores de diversidad, de competición, de éxito individual; y tolera benevolentemente, además, ciertas prácticas de explotación del trabajo, de evasión fiscal y contributiva, e incluso de pequeña picaresca. Está claro que se trata de valores y actitudes ajenos, cuando no antagonistas, a la naturaleza y filosof́a del sindicato, el cual privilegia valores de igualdad y solidaridad, de conflicto de grupo y entre intereses organizados, de elevación social colectiva, de reguslación del mercado, de limitación de la intensidad del trabajo, de lealtad fiscal (porque el Estado constituye un valor positivo, y porque el trabajo dependiente no puede eludir el fisco). El éxito del sistema de la pequeña empresa actúa de tal modo que, en los años orhenta, los valores de los que se hace estructuralmente el portador se difunden socialmente, afimándose a nivel de masas: esto significa, para el sindicato, la difusión de ideas e ideales ajenos y, por tanto, implica la necesidad de ir a contra corriente en medio de los obstáculos de un sistema de valores que se opone culturalmente a su expansión. 


\section{LAS TRANSFORMACIONES EN CURSO}

A partir del cuadto que hemos descrito, intentemos ahora identificat más precisamente cuáles y cuántos cambios han acontecido en la sociedad italiana de estos años, viendo cómo ésos modifican la situación de contexto del actor sindical. Procederemos en forma de tipificación ideal, adoptando como hipótesis de partida una serie de recorridos desde un punto A hasta un punto $B$ de la situación e ilustrando sus consecuencias.

El primer paso, discutido por muchos (Pizzorno, 1980; Accorneto, 1981), parte de la figura sociológica del obrero-masa, que se encuentra en el centro del proceso productivo durante toda una fase del desarrollo industrial (hasta mediados de los años setenta) peto conoce un declive progresivo en cuanto a su presencia cuantitativa e impottancia cualitativa. Aquello que para el sindicato había sido el sujeto social mayormente representado se desplaza hacia los márgenes de la escena productiva, dejando campo libre a la figura del obrero-individuo - y por otro lado, como lo veremos, a la figura del individuo-familia, para decirlo como Schumpeter (1927). Si bien el sindicato conserva una amplia aceptación en la nueva clase obrera, la relación entre base y organización entra, sin embargo, en una crisis objetiva. La organización, tanto en sus cuadros dirigentes, en su ideología, como en sus opciones políticas y negociadoras, se encuentra cada vez más a menudo en una situación de no correspondencia respecto a los sujetos concretos que constituyen su base. Por vía de una comprensible inercia, el grupo social de referencia del sindicato tiende a seguir siendo el obreromasa, con sus necesidades, sus intereses y su visión del mundo. Con dos consecuencias: una, el deslizamiento progresivo de la representación desde el centro hacia la periferia del sistema, a temolque, por así decirlo, de una clase, la obrera de la gran industria, que va perdiendo importancia cuantitativa y cualitativa; otra, la incapacidad progresiva de representar los intereses, las necesidades y los valores nuevos que van afanzándose en la propia base y - todavía más-n en los grupos sociales y profesionales que se manifiestan reclamando una nueva representación. Por ello, tiende a estabilizarse una situación de bias estructural entre organización sindical y mundo del trabajo, con una fuerte capacidad de representación en los sectores en receso o en crisis y una débil en los nuevos y más dinámicos (Cesos, 1988).

E1 segundo paso puede teducirse, como tipificación ideal, al binomio empleo singular - pluriempleo, mediante el cual se señalan transformaciones de género diverso. Una es la introducción cada vez más masiva del principio de variabilidad en las condiciones laborales, que sustrae progresi- 
vamente al trabajador a la inmovilidad ocupacional, desplazándolo frecuentemente, a lo largo del ciclo vital, de sector en sector y de oficio en oficio. La segunda es la difusión de una pluralidad de formas de relación laboral que redimensiona objetivamente la hegemonía del trabajo estable, organizado, de duración estándar. Sobre todo, pero no exclusivamente, en los estratos nuevos de la población labotal tienden a ganar terreno unas formas de trabajo a tiempo parcial, a plazo fijo, de formación y trabajo, y otras parecidas (Acconnero, 1986), con un replanteaniento de la división tradicional entre trabajo y no trabajo, sea en el transcurso del día como en el de la vida, y con una amplia introducción del principio de alternancia y de discontinuidad (por ejemplo, entre trabajo y estudio, pero también entre tiempo libre y trabajo, entre trabajo y trabajo, etc.). En tales condiciones, la acción sindical se hace estructuralmente más difícil, debido a Ia dificultad aumentada de organizar intereses y necesidades tan diversos y tan diversamente presentes en el lugar de trabajo (Sutrenti, 1981), pero también debido a la costumbre cultural e ideológica de refetirse al trabajo como un hecho organizativo totalizador de Ia jornada y de la vida del trabajador, cuando por el contratio se encuentran en ellas, cada vez más a menudo, articulaciones y complejidades.

El tercer aspecto puede describirse como un teplanteamiento de la categoría del trabajo dependiente (con su base individual) en beneficio del in. dependiente (con su base familiar). En este caso, más que referimos a las transformaciones en las respectivas proporciones (Brunetta-Dal, 1987), quezemos indicar aquí como los más recientes desarrollos han sumado indeterminación a la categoría del trabajo dependiente, cada vez menos identificable con la suficiente precisión sociológica. Las razones son de varios tipos. La mezcla de roles autónomos, semiautónomos y dependientes en el interior del mismo núcleo familiar - una realidad hoy en día bastante difundida (David. Vicarelli, 1983) - impide una clara colocación objetiva y subietiva en la estratificación social de los trabajadotes individuales. Si el núcleo de base de la estructura social es el individuo-familia (lo que parece sociológicamente correcto), la representación de los intereses se vuelve excesivamente problemática, dada la inevitable mediatización que se produce en el interior de una familia, en la que los roles laborales son socialmente compuestos. Para el trabajador individual, los intereses representables llegan a perder solidez y claridad y, por tanto, falla la posibilidad de una delegación sin reservas en manos de la organización sindical.

En el caso no infrecuente en que la «promiscuidad» ocupacional se presenta en un mismo trabajador, Ias evaluaciones mencionadas más arriba resultan enfatizadas. Nos referimos aquí a la fuerte difusión de las figuras mixtas, tales como las que combinan en una misma persona deberes labora- 
les y situaciones profesionales de diverso típo: toda la literatura dedicada al doble empleo ofrece una amplia castústica sobre el tema (Gallino, 1985). Desde nuestro punto de vista, queda clara la mayor debilidad de la representación sindical, ya que el trabajador dependiente puede ser también (en su segundo trabajo) un trabajador autónomo, o porque, de cualquier forma, la combinación de dos o más trabajos induce a unos comportamientos (tales como la evasión fiscál y contributiva) condenados en la afiliación sindical; y no bablemos, obviamente, de la reprobación social hacia el pluriempleo que, con su postura, va en contra de uno de los valores fundamentales del credo sindical en Italia: la solidaridad hacia quien no tiene trabajo (Cella, 1981).

Finalmente, se pueden realizar evaluaciones análogas acerca de aquelios trabajadores que, por diversos motivos, no poseen una situación social y sindical clata y automática. Éste es el caso, sobre todo, de los artesanos y, en general, de los trabajadores por cuenta de terceros que, genéricamente, son independientes pero se hallan a menudo en condiciones de dependencia sustancial respecto a sus compradores. Su origen social, a la par que la manualidad de sus tareas, los acercan ciertamente al trabajador dependiente; pero no escapará a nadie la atracción social e ideológica que puede ejercerse sobre ellos bajo la influencia del carácter microempresarial de su rol, de su independencia y autonomía de decisión, de su relación con el «libre» mercado, etc.

En cierta medida, estas valoraciones resultan también extensibles a los trabajadores dependientes de pequeñas empresas y pequeñas sociedades de servicios. Muchas investigaciones sobre el tema (Bagnasco.Triglia, 1984 y 1985) han señalado una participación muy particular por parte de tales trabajadores en el destino de la empresa, ya que se encuentran trabajando hombro con hombro con su "jefe» (a menudo un ex trabajador $o$, de cierta manera, de extracción social homogénea con la de los mismos trabajadores dependientes), y compartiendo a veces su suerte mediante unas formas de incentivos salariales ligados a la marcha de los beneficios (éste es el caso, sobre todo, de las pequeñas empresas de servicios en las que no es raro ver como los asalariados llegan a verdaderas formas de participación en los beneficios). Para estas categorías, que objetivamente son de trabajadores dependientes, las difcultades de representación sindical no solamente constituyen un elemento objetivo ligado a las pequeñas dimensiones, sino a menudo también un elemento subjetivo, dada la fácil identificación entre el trabajador y «su» empresa, entre el asalariado y su «jefe». Esto explica, y ya hemos aludido a ello, por qué la lógica de la afiliación sindical, y más todavía la del comportamiento conflictual, resultan, en tales áreas laborales, bastante diferentes de la de la gran empresa y tienden a manifestarse como un antagonismo respecto al exterior antes que con respecto al interior de 
la empresa y dirigido más hacia lo "público» (las organizaciones empresariales, los gobiernos locales y nacionales) que hacia lo "privado» (el empresario en singular) (Trigilia, 1985).

El cuarto aspecto destaca una transformación que se desenvuelve toda ella en el plano cultural y en el de los valores, y que realza un juego complejo de interacciones entre las transformaciones de la estructura productiva, ocupacional y de la organización del trabajo, y los cambios en el sistema de valores y en las actitudes de las capas sociales involucradas en ellas. En sustancia, estamos en presencia del paso desde una situación en la que prevalece el trabajo objetivamente determinado hacia otra catacterizada por el trabajo subjetivamente buscado. Se hace notar con prepotencia una larga serie de efectos: los de la escolarización de masas, que hace que todos estén más sensibles y al tanto de sus propias exigencias y necesidades (también en términos de realización personal); los de los movimientos colectivos de finales de los sesenta y primeros setenta, que han dejado en herencia a ciertos grupos sociales y generacionales (sobre todo mujeres y jóvenes) un inquieto patrimonio de libertad, autonomía y capacidad crítica; y finalmente, los de un crecimiento civil y cultural que penetra tanto en Italia como en todos los países europeos a partir de los años setenta y que lleva a amplias franjas de la pobiación, en especial en sus áreass más modernas, a desarrollar unas orientaciones de valor más decisivamente "posmaterialistas» (Inglehatt, 1977; Giovannini, 1982), menos fácilmente adaptables respecto a la situación existente. Por lo que respecta a nuestro análisis, esto implica una inversión parcial de la óptica interpretativa tradicional, que ve cómo la dinámica estructural modela según sus propias exigencias el mercado del trabajo y los sujetos (individuales y sociales) que en él se presentan. En realidad, los años recientes han indicado con claridad que existe un espacio autónomo creciente de intervención de la oferta laboral que termina por influir fuertemente las configuraciones concretas de una situación ocupacional, sus formas organizativas y hasta (hipotéticamente) los caracteres locales de una formación social. Es probable que, en cierta medida, siempre haya ocurrido así y que cualquier modalidad de determinación «desde abajo» se encuentre entre las causas no últimas de la elección de una vía u otra hacia el desartollo del capital. Peto hoy en día tales influencias parecen bastante más relevantes: es razonable considerar, por ejemplo, que el tápido crecimiento del sector tetciario público y privado en Italia no tiene solamente el sentido relativamente común de una dificultad en $\mathrm{l} l$ desarrollo industrial, sino que es también el fruto de un empujón en esta dirección por parte de los com. ponentes sociales y generacionales que buscan en el trabajo, en este trabajo, aquelia congruencia respecto a sí misnos y a la propia formación que sabian no poder encontrar en el sector de la industria. El desarrollo mismo 
de la pequeña empresa, debido al espacio que deja a la iniciativa e inventiva personal, y debido también a la menor impersonalidad de las relaciones sociales y de trabajo, a las posibilidades de movilidad que pernite y a una cierta lógica cooperativa que conlleva estructuralmente (de cooperación interna dentro de la empresa y de cooperación entre empresas), parece corresponder mejor a las nuevas subjetividades e incluso, quizás, representat su expresión, tal como parece indicarlo el elevado grado de consenso que acom. paña a menudo, incluso entre las nuevas generaciones, tal forma de desarroIlo (Capecchi-Pesce, 1983). Para el sindicato, esta mayor incidencia de la subjetividad indivioual en el campo laboral (lo cual quiere decir: en el lugar y el modo en que se busca, se encuentra y se realiza un trabajo) aumenta las dificultades de representación, porque disminuye la uniformidad y aumenta las diferencias, porque vuelve menos previsibles los flujos ocupacionales y las dinámicas profesionales, porque obliga a una organización de masas (culturalmente acostumbrada a tazonar en términos de determinismo económico) a tener en cuenta unas lógicas nuevas y unas variables independientes que corresponden a unas subjetividades individuales, de grupo y de oficio. No por azar es entre los jóvenes donde el sindicato encuentra las mayores resistencias, tanto respecto a la afiliación, como (o más) respecto a la militancia sindical (Giovannini, 1985): de tal modo que se plantea hoy día, para el sindicato, un dramático problema de reproducción social sin precedentes en toda su historia.

El quinto y último punto que debo señalar concierne el sistema de las desigualdades. Se sabe que el sindicato crece y modela su organización en el lugar de trabajo, a partir de las numerosas desigualdades verticales que nacen de una relación de producción y organización del trabajo. Históricamente, esto ha conducido a privilegiar aquellos grupos relativamente amplios y telativamente homogéneos (campesinos, obreros, empleados, técnicos, etc.) que definían su postura con teferencia a las condiciones de trabajo, y que se encontraban en situación de desarrollar unas capacidades organizativas y de generar unos comportamientos conflictivos (Off́e, 1977). Se ha prestado poca atención a los intereses y a las necesidades que se crean fuera del trabajo y le son ajenas, y que guardan más relación con el problema de la convivencia social (desde Ia vivienda hasta los servicios sociales y sanitarios, desce la educación al tiempo de ocio, etc.). En tales áreas, ajenas al mercado y a la distribución, es donde se van creando sistemas de desigualdades borizontales que aúnan (o contraponen) a los grupos sociales, las clases, las franjas de edad, las generaciones, etc., independientemente (y, por así decirlo, transversalmente) en las confrontaciones de las condiciones de trabajo respectivas. El nuevo sistema de desigualdades se forma especialmente en aquella área de densa convivencia social que es la ciudad (y la me- 
trópoli), genera más fácilmente los movimientos colectivos que los fenómenos de organización o, como mucho, organiza sus propias representaciones según la lógica de la single-issue politics (Melucci, 1986). Para el sindicato, la extensión de las desigualdades horizontales comporta una inevitable crisis de representatividad, pero, sobre todo, impone una transformación radical de los objetivos y de las estructuras organizativas, con el fin le propiciar la capacidad de movilización y de representación incluso sobre el territorio (y especialmente en las zonas urbanas), además del lugar de trabajo; y con el fin de permitir la confrontación con las nuevas contrapartes puestas en evidencia por el nuevo sistema de desigualdades (poderes públicos, organizaciones que asumen los intereses, etc.).

Trataremos separadamente un último aspecto de la crisis de representación porque atañe a unas transformaciones que sólo conciernen indirectamente al sindicato. Me refieto al desapego general hacia la política que parece caracterizar la sociedad italiana después de la gran crisis de la segunda mitad de los años setenta, y cuyo momento más dramático ha sido el de la trágica experiencia terrorista. En el plano político, la señal más evidente de tal desapego ha sido el fuerte crecimiento de la abstención electoral a partir de las elecciones políticas de 1979 (Caciagli-Scaramozzino, 1983), pero también la caída de la participación política y de la afliación a los partidos, especialmente entre las nuevas generaciones (Caciagli, 1987; Giovannini, 1988). Es probable, dada la naturaleza política del sindicalismo italiano, que los efectos de tal desapego hayan afectado también a los sindicatos confederales, disminuyendo la propensión a participar y afiliarse. Es probable, también, que Italia se resienta, desde este punto de vista, de las mismas tendencias a la baja en la participación que ya han afectado, desde hace tiempo, a los países más avanzados y especialmente a los Estados Unidos.

Aun cuando no pretenda volver sobre un debate propiamente politoló. gico, quisiera señalar aquí los aspectos del fenómeno que atañen más directamente a la problemática discutida y a su especificidad nacional. El primer aspecto concierne la transformación de la peculiax relación de crédito que ha ligado tradicionalmente los trabajadores italianos a sus sindicatos, y especialmente a la CGIL y a Ja CISL. En las vastas áreas territoriales del centro de Italia (de cultura prevalentemente comunista) y del nordeste (de cultura prevalentemente católica), la adhesión a los sindicatos tespectivos ha sido, hasta los años recientes, un hecho «natural», de identificación automática entre base y organizaciones de representación (sea tanto política como sindical) (Trigilia, 1986). Las razones de la adbesión residían en el sentido de pertenencia típico de toda subcultura (territorial o de clase), que hacía particularmente sólido y resistente en el tiempo el lazo de unión con las organizaciones propias (Parisi-Pasquino, 1977). La disgregación de las sub- 
culturas políticas, que se va notando desde los años setenta bajo la intuencia modernizadora de la escolarización y de la cultura de masas, transforma silenciosamente los mecanismos de la adhesión sindical, especialmente entre las nuevas generaciones: primero, debilitando los procesos de socialización política de una generación a otra; segundo, reduciendo drásticamente la necesidad de expresar los propios puntos de referencia (debido a la afrrmación de una concepción laica de la convivencia social que hace menos apremiante la reclamación de la propia identidad); tercero, haciendo surgir una fuerte tendencia a la participación más dictada por la lógica de los intereses que por incentivos de identidad (Pizzorno, 1980). Para el sindicato, las consecuencias de todo ello no sólo se miden en una caída general de la participación, sino también en una mayor incertidumbre de la adhesión, dado que las nuevas modalidades (de intereses o de opinión) son bastante menos pegadizas que las anteriores (de identidad o pertenencia), con la consecuencia de una mayor movilidad entre afiliación y no afiliación, y entre afiliacio. nes a un sindicato u otro. En resumidas cuentas, aquello de estar afliado a un sindicato es cada vez menos un "valor" considerado como positivo, e incluso entre los jóvenes parece ir afirmándose un «contravalor» respecto a las confrontaciones de la afiliación sindical (Signoti, 1988).

Naturalmente, no siempre resulta fácil evaluat en qué medida esta caída en la demanda de señas de identidad es debida a razones de orden general y en qué se debe a motivos de orden específico. De hecho, es indudable que las sociedades contemporáneas ofrecen, hoy en día, un más amplio repertorio de lugares de oferta y conformación de identidad ajenos al trabajo y al sindicato; y que, por tanto, debilitan objetivamente las chances de éxito de aquellas organizaciones fundadas y centradas en el tiempo dedicado al trabajo. Pero también es cierto que la oferta de señas de identidad por parte del sindicato se ha vuelto más problemática e incierta, y no ha sido sustituida por una capacidad igualmente eficaz de representación y defensión de los intereses y necesidades de su base potencial. Además, poco se ha hecho para hacer frente a las transformaciones acontecidas en el plano cultural y de la comunicación, de tal modo que el sindicato, hoy, resulta también por eilo más ajeno aún a la mismísima sociedad y en especial a sus componentes juveniles. Un aparato simbólico, un lenguaje y unas modalidades de comunicación entre sindicato y base, hoy por hoy generalmente inmutables (Ambrosi, 1987), diferencian culturalmente al sindicato de las entidades competidoras y lo hacen poco sintónico con las nuevas culturas: tanto más que se ha derrumbado, si es que alguna vez existiera, el monopolio de la información acerca de los problemas labotales y que se entremezclan las antiguas estrategias de comunicación con las redes informativas nuevas y sin prejuicios de los mass-media (Bianchi, 1988). 
EI sindicato en Italia: ¿una crisis de representación?

\section{CONCLUSIONES}

En estas páginas, mi propósito se ha limitado deliberadamente a señalar, entre las numerosas causas posibles de la crisis actual del sindicato, las que pueden relacionarse con un estado no tanto patológico como fisiológico de crecimiento y desartollo de la sociedad italiana, rehuyendo implícitamente una interpretación coyuntural de la presente fase bistórica, según la cual estará sucumbiendo, o casi, la vieja y conocida sociedad industrial debido al empuje de la revolución tecnológica, de la reestructuración industrial y terciaria, y de las transformaciones culturales y generacionales. Me parece que poco sentido tiene, si es que alguna vez lo tuviera, proponer una lectura del desarrollo social en términos rígidannente deterministas. Aun cuando fuera verdad, y probablemente lo es, que hoy en día nos hallamos en la fase creciente de una nueva etapa del desarrollo, viendo cómo las fuerzas en presencia se van ordenando bajo una suteva organización del trabajo productivo y social, con todo, no podemos pensat tranquilamente en unas estrategias de tipo adaptativo como las únicas posibles. Andat a temolque de las transformaciones sociales, tal como parece que ha ocurrido hasta hoy, Lleva inevitablemente a unas transformaciones de signo negativo en la estructura, naturaleza y acción del sindicato. Tanto el crecimiento del peso porcentual de las afiliaciones en el Sur y su declive en el Norte (Regalia, 1981) como la fuerte presencia de categorías marginales (sobre todo los jubilados), acompañada por la caída de los afiliados entre los trabajadores de la industria (cf. Ias tablas 1-2), terminan por derivar el sindicato bacia corrientes poco fructíferas: por una parte, tedefiniendo sus tateas en un sentido asistencial, de prestación de servicios a unas categorías débiles (como, por ejemplo, los jubilados) (Donolo, 1981); y, pot otra parte, impulsándolo a una fuga en el sentido institucional (Regini-Regalia, 1982) que si, por un lado, permite afrontar los aspectos más aparentes de la crisis (precisamente a través de una presencia sindical en las instituciones), por otro lado, lo aleja todavía más de los problemas e intereses concretos de su propia base $y$, por tanto, de sus posibilidades de reproducir y extender su propio consenso (Romagnoli, 1980).

En realidad, toda transformación debería ser considerada como un desafío al cual fuera menester dar tespuesta en los propios términos, lo que quiere decir, en el caso del sindicato, en términos coherentes con su propio proyecto de plena valoración de los intereses y de los valores de aquellos a quienes representa o aspira a representar. De otro modo, otras fuerzas sa. brán encontrar unas áreas de consenso en relación a sus proyectos, explícitos o implícitos, tal como, en parte, ya demostraron que saben hacerlo. 


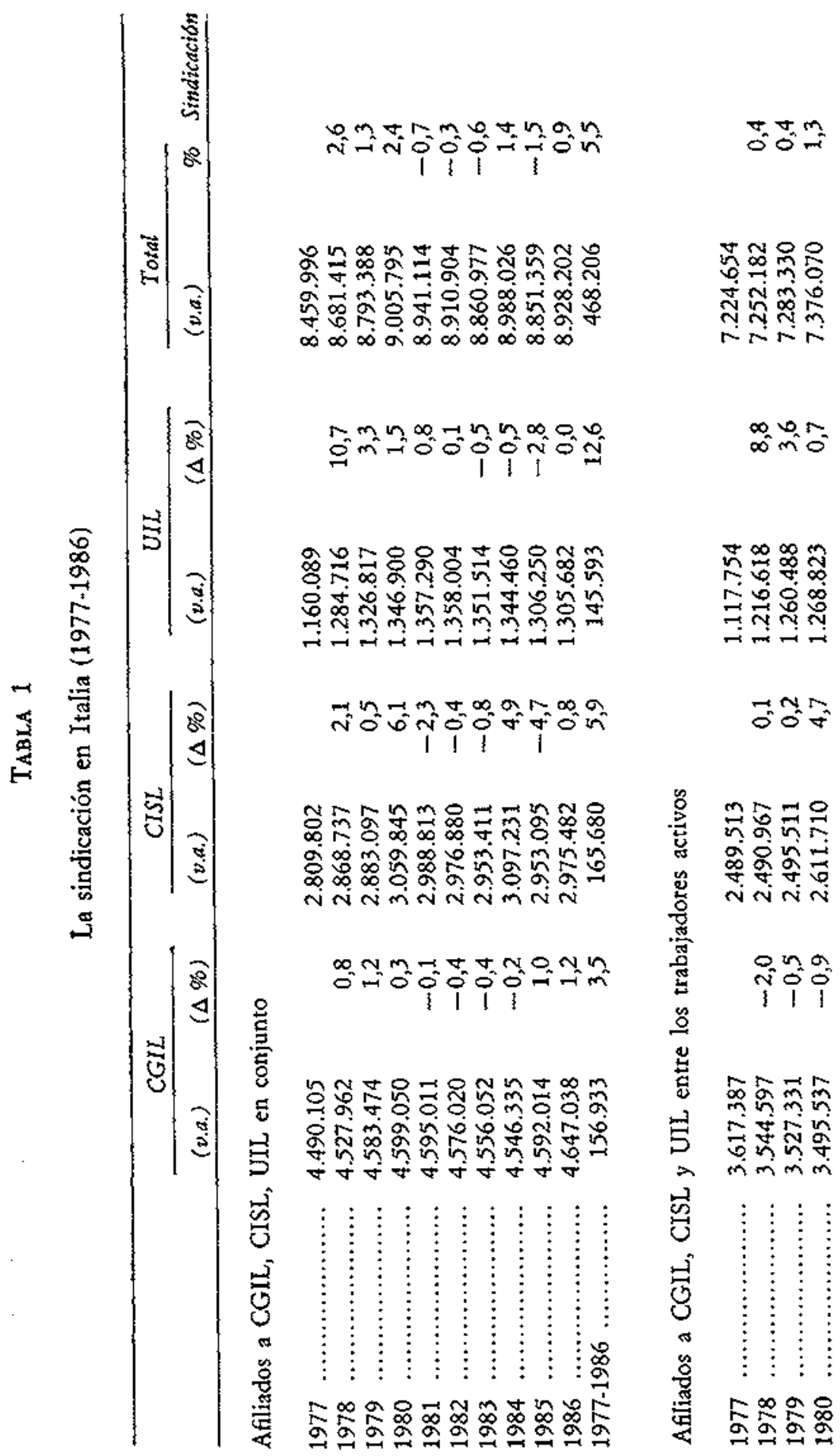




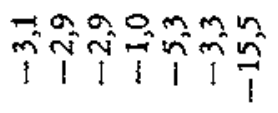

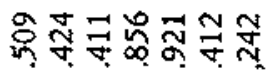
廿 Nob $060 \%$

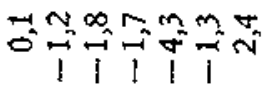

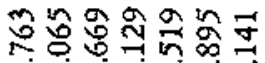

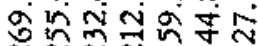

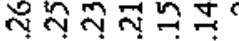
मां

$\Rightarrow a \Rightarrow+20$ जीNNक $\begin{array}{lllll}1 & 1 & 1 & 1\end{array}$

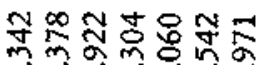

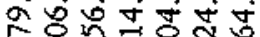

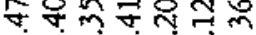
NกN

$\infty$ in o m o $\infty$ m

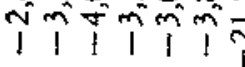

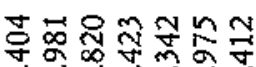

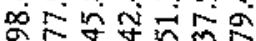

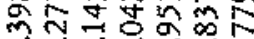
mimmin

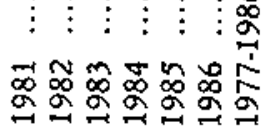

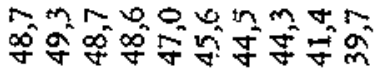

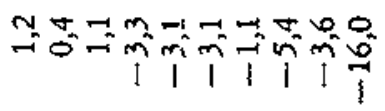

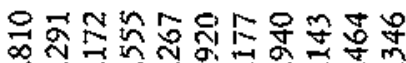

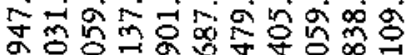

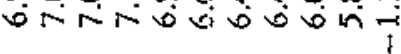

$$
\text { nून }
$$

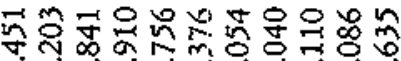

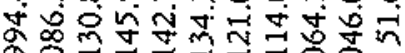

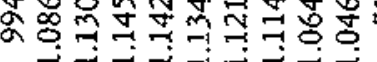

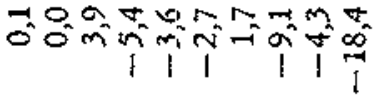

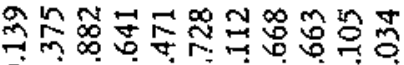
ำง ชาㄱำ

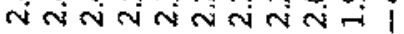

$$
\text { mas }
$$

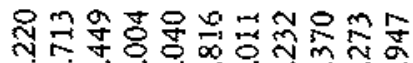

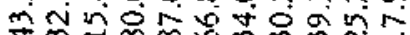

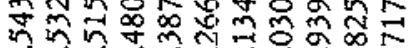

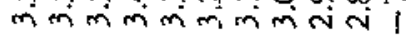




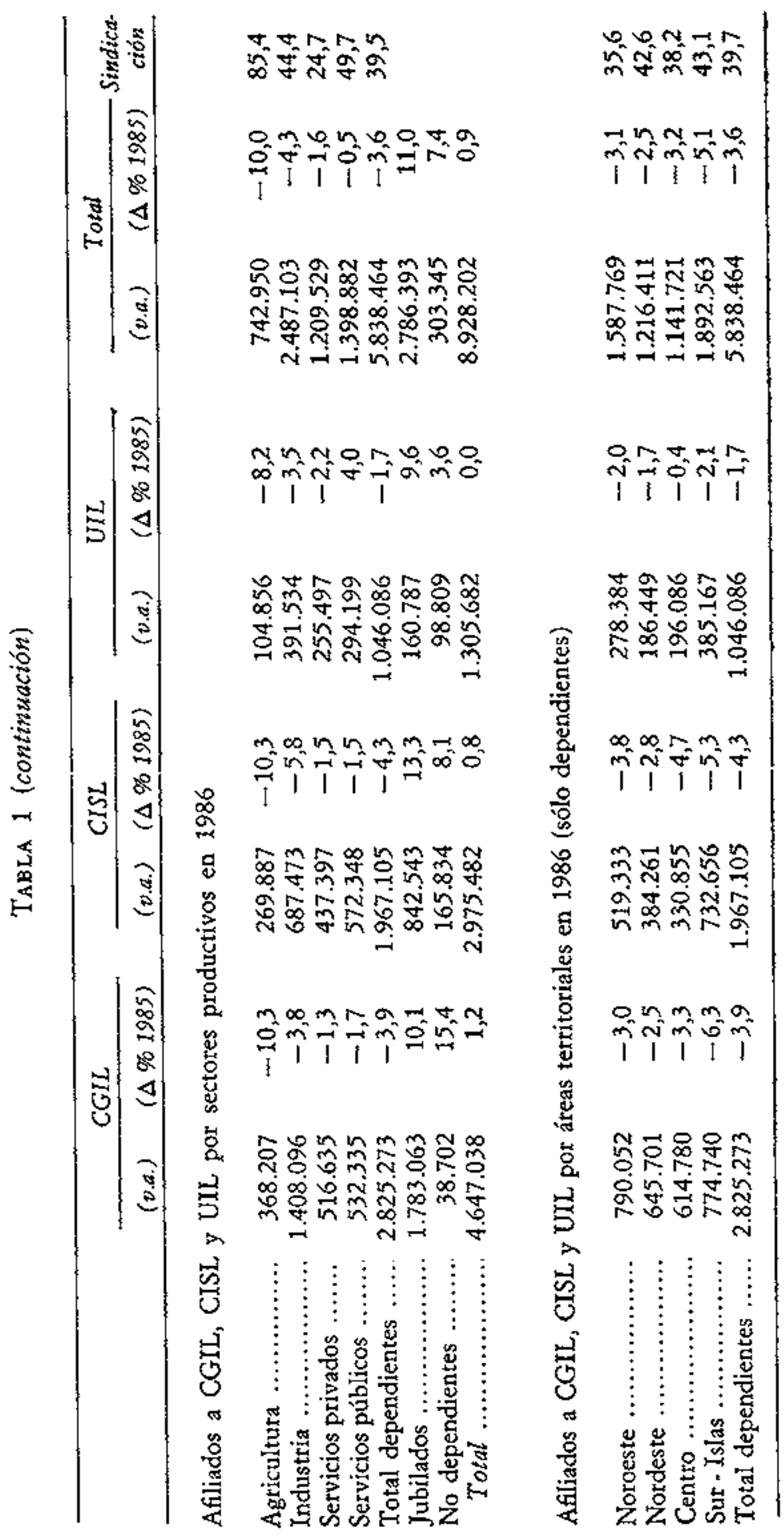


TABLA 1 (continuación). En porcentajes.

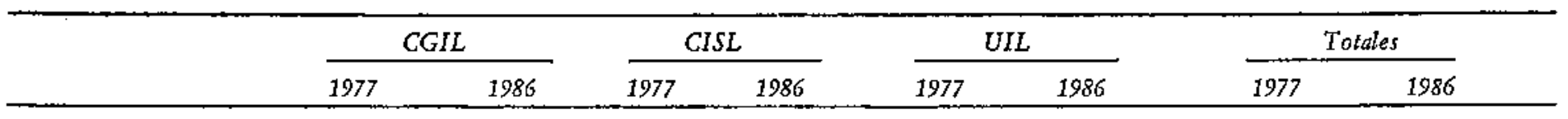

Contribución de cada confederación a la sindicación por áreas territoriales (sólo dependientes)

\begin{tabular}{|c|c|c|c|c|c|c|c|c|}
\hline Noroeste ................ & 52,3 & 49,8 & 35,0 & 32,7 & 12,7 & 17,5 & 100 & 100 \\
\hline Nordeste ................. & 55,1 & 53,1 & 32,0 & 31,6 & 12,9 & 15,3 & 100 & 100 \\
\hline Centro $\ldots \ldots \ldots \ldots \ldots \ldots, \ldots$ & 56,9 & 53,8 & 28,9 & 29,0 & 14,2 & 17,2 & 100 & 100 \\
\hline Sur - Islas ............... & 43,2 & 40,9 & 39,8 & 38,7 & 17,0 & 20,4 & 100 & 100 \\
\hline Total dependientes ....... & 51,0 & 48,4 & 34,7 & 33,7 & 14,3 & 17,9 & 100 & 100 \\
\hline
\end{tabular}

Peso porcentual de las áreas territotiales en cada confederación y sobre el total (sólo dependientes)

\begin{tabular}{|c|c|c|c|c|c|c|c|c|}
\hline Noroeste $, \ldots \ldots \ldots, \ldots \ldots$ & 31,0 & 28,0 & 30,5 & 26,4 & 26,8 & 26,6 & 30,2 & 27,2 \\
\hline Nordeste ................. & 22,9 & 22,9 & 19,5 & 19,5 & 19,1 & 17,8 & 21,2 & 20,8 \\
\hline Centro $\ldots \ldots \ldots \ldots \ldots \ldots$ & 20,6 & 21,8 & 15,4 & 16,8 & 18,3 & 18,7 & 18,5 & 19,6 \\
\hline Sur - Islas ................. & 25,5 & 27,4 & 34,6 & 37,2 & 35,8 & 36,8 & 30,1 & 32,4 \\
\hline Total dependientes ...... & 100,0 & 100,0 & 100,0 & 100,0 & 100,0 & 100,0 & 100,0 & 100,0 \\
\hline
\end{tabular}

FURNTE: CESOS, Le relazioni sindacali in Italia: Rapporto 1986-1987, Roma, 1988. 


\section{TABLA 2}

La sindicación por sectores (1981 y 1986)

Confrontación entre 1981* y 1986 de los afiliados a SGIL, CISL y UIL en conjunto, por sectores de pertenencia e incrementos porcentuales

\begin{tabular}{|c|c|c|c|c|c|c|c|c|c|c|c|c|}
\hline \multirow[b]{2}{*}{ Sectores } & \multicolumn{3}{|c|}{ CGIL } & \multicolumn{3}{|c|}{ CISL } & \multicolumn{3}{|c|}{ UIL } & \multicolumn{3}{|c|}{ Total CGIL, CISL y UIL } \\
\hline & 1981 & 1986 & var. \% & 1981 & 1986 & var. $\%$ & 1981 & 1986 & var. $\%$ & 1981 & 1986 & var. \% \\
\hline Agricultura ............... & 519.028 & 368.207 & -29 & 485.229 & 269.887 & $-44,4$ & 166.310 & 104.856 & $-36,9$ & 1.170 .567 & 742.950 & $-36,5$ \\
\hline Industria $\ldots \ldots .$. & 1.757 .954 & 1.408 .096 & $-19,9$ & 950.560 & 687.473 & $-27,7$ & 481.947 & 391.534 & $-18,7$ & 3.190 .461 & 2.487 .103 & -22 \\
\hline Terciatio........... & 1.121 .422 & 1.048 .970 & $-6,5$ & 1.043 .553 & 1.009 .745 & $-3,2$ & 540.160 & 549.696 & 1,8 & 2.705 .135 & 2.608 .411 & $-3,6$ \\
\hline Jubilados ** $\ldots . . . \ldots \ldots$ & 1.186 .207 & $\begin{array}{c}1.821 .765 \\
(38.702)\end{array}$ & 53,6 & 509.471 & $\begin{array}{l}1.008 .377 \\
(165.834)\end{array}$ & 97,9 & 168.873 & $\begin{array}{r}259.596 \\
(98.809)\end{array}$ & 53,7 & 1.864 .551 & $\begin{array}{l}3.089 .738 \\
(303.345)\end{array}$ & 65,7 \\
\hline Totales & 4.584 .611 & 4.647 .038 & 1,3 & 2.988 .813 & 2.975 .482 & $-0,4$ & 1.357290 & 1.305 .682 & $-3,7$ & 8.930 .714 & 8.928 .202 & $-0,1$ \\
\hline
\end{tabular}

* Datos sobre 1981 extraídos del Rapporto CESOS 1981, mientras que los de 1986 del de 1986/1987.

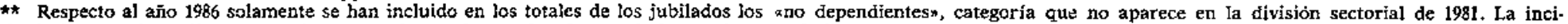
dencia de los «no dependientes resulta, con todo, modesta y los datos relativos a éstos se indican entre paréntesis debajo del total general de los jubilados.

FuEN̦IE: CESOS, Le relazioni sindacali in Italia: Rapporto 1981, Roma, 1982. CESOS, Le relazioni sindacali en Italia: Rapporto 1986/1987, Roma, 1988. 
¿No nos encontramos acaso en presencia de los vistosos éxitos de tales estrategias ajenas cuando medimos el elevado grado de consenso (o de no oposición) que acompaña a unos procesos tan costosos como penosos de reestructuración, de redistribución anárquica del trabajo y no trabajo, de flexibilidad incontrolada y unilateral? No se puede menos que pensar que la explicación de tales éxitos se halla en una correspondencia, al menos patcial, de las estrategias adoptadas con unas subjetividades nuevas y poco conocidas, difundidas tanto entre los trabajadores «tradicionales» como entre los «modernos», entre ocupados y parados, entre jóvenes y menos jóvenes. Sujetos y subjetividades que evidentemente escapan al sindicato, en el doble sentido de la palabra: por un extrafiamiento creciente de los estratos y grupos sociales respecto a la institución sindical, ya no buscada sino incluso rehuida; y por una inadecuación estructural, organizativa y cultural por parte del sindicato para llegar a conocer en sentido profundo lo que está cambiando y ha cambiado en la sociedad italiana, y específcamente en aquel universo cada vez menos uniforme que es el trabajo dependiente.

Hemos Ilegado así a la cuestión de fondo. El sindicato, en tanto en cuanto institución y sujeto autónomo, puede seguir existiendo y ejerciendo su propio papel mientras conserve o renueve aquel bien específico que constituye el conseraso teal y pleno de los propios tepresentados. En definitiva, sólo esto legitima su acción y le proporciona fuetza y espacio, tanto de cara al sisterna político como de cara al sistema de las empresas. Si la vieja tepresentación se ha ido consumiendo, es necesario que el sindicato se prepare rápidamente a representar la nueva, para que la crisis en curso sea de transformación y no de deciive. Entonces, ¿en qué terrenos emprender los primeros pasos? Si el esbozo de análisis propresto aquí tiene alguna validez, son tres, a mi parecer, y en estrecha relación mutua, los problemas que deben afrontarse con prioridad:

1) crecimiento de la capacidad cognoscitiva; 2) reelabotación de los cimientos culturales; 3) experimentación organizativa. En cuanto a la necesidad de realizar tápidos pasos hacia adelante en el primer terreno, no hay dudas posibles. La madurez individual y colectiva en el terreno del conocimiento significa la posibilidad fundada, racional e institucional $-\mathrm{y}$ no episódica o voluntarista- de enfrentarse válidamente, partiendo de bases nuevas, tanto con el poder empresarial como el político, y de recuperar conocimientos, informaciones y capacidad de decisión: en una palabra, de volver a encontrar fuerza de proposición, autonomía e independencia real. Una condición previa más importante es el «desenvejecimiento» de la mentalidad sin. dical a favor de una fuerte y libre circulación de ideas y hombres, a favor de la desaparición de las rigideces organizativas e ideológicas persistentes, a favor de una franca aceptación de la confrontación con todo cuanto se de- 
bate y se expresa mientras sea nuevo, incluso si esto significa poner en entredicho o llevar a debate los pattimonios tradicionales de pensamiento y acción.

El segundo problema, el de una reelaboración de los cimientos culturales del sindicato, es tan urgente como el primero. La cultura sindical está, hoy por hoy, ampliamente fundada en una visión colectiva, de masas, y relativamente rígida, de los sujetos y de los intereses representables. Ésta se va adaptando con creciente dificultad a una sociedad objetiva y subjetivamente diferenciada, que impone la adopción de más numerosas estrategias, de modelos de acción y organización sindical articulados (Regini, 1988). Se hace cada vez más patente la sensación de que el sindicato, no sólo y no tanto debido a las dificultades de la situación sino, más que nada, debido a sus adherencias ideológicas y culturales, logra cada vez menos dirigir las transformaciones en curso y se demora en negociar el cuánto y el cómo de su retirada; o, para ser menos drásticos, se mantiene parado en plan de resistencia y defensa ante las medidas tomadas por otros en otra parte (las empresas y el Estado, en primer lugar; pero incluimos también la fuerza más «neutral» de la innovación tecnológica).

Lo que hace falta, ahora, es la transición hacia un nuevo modelo organizativo y cultural (en cierto sentido, hacia un nuevo sindicato) que proporcione tanto instrumentos como imaginación para poder pasar de la «resistencia» a la negociación -0 , mejor dicho, a las negociaciones: aprovechando la - ocasión de la flexibilidad exigida por las nuevas tecnologías y, por razones menos lineales, por las empresas, para hacer valer los intereses y necesidades diversificados que hoy en dia son apenas o nada representados por el sindicato--, en definitiva, para hacer valer las exigencias no menos importantes de variabilidad y flexibilidad de los trabajadores.

Finalmente, el tercer problema. Es adrede que no se intenta proponer aquí operación de ingeniería organizativa alguna, sino, más pragmáticamente, una apertura programada hacia la experimentación y la innovación. Inicialmente, éstas podrían probarse en unos espacios y tetrenos relativamente circunscritos - definidos, por ejemplo, por las problemáticas que éstos afrontan y la instrumentación contractual que utilizan-, peto de manera que funcionen como un canal o punto de paso a través del cual hacer llegar al sindicato unas ideas y experiencias no tradicionales, que sean un lugar de elaboración prospectiva y un termómetro sensible a las transformaciones en curso, y que se concretan en centro y ocasión para la selección y formación de un leadersbip renovado en su mentalidad y en sus modalidades de acción (Manghi, 1981; Pasquino, 1983; Regalia, 1986). Los lugares de la moderna convivencia, las cíudades y grandes urbes del desarrollo, con sus desigualdades, las viejas y las nuevas, padrían constituir el terreno sobre el cual po. 
El sindicato en Italia: ¿una crisis de representación?

nerse a prueba y renovarse a través de la defensa de unos derechos y de la proposición de unos intereses hoy en día poco o nada representados. Este es un camino que requiere unas energías y una mentalidad renovadas, pero que quizá presenta pocas otras alternativas para el sindicato que no sean la vía del declive o la transformación en un sentido corporativo. 


\section{BIBLIOGRAFIA}

Accornero, A. (1986): Il sindacato nella società flessibile, en Il futuro del sinda. cato, Ferrante, G., Roma, Ediesse.

- (1981): «Sindacato e rivoluzione sociale. Il caso italiano degli anni 70», Laboratorio politico, núm. 4.

Ambrosi, E. (1987): Nella misura in cui... Il linguaggio sindecale, Roma, E. Lavoto.

Antoniazzi, S. (1986); «Il militante e la sua identità», Prospettive sindacali, número 57.

- (1986): La solidarietà, la lotta, la cultura, Roma. Ed Lavoro.

Ascoli, U. y Catanzaro, R. (1987): La società italiana degli anni ottanta, Bari, Laterza.

Bagnasco, A. (1977): Tre Italie, Bolonia, II Mulino.

- (1988), La costruzione sociale del mercato, Bolonia, I1 Mulino.

Bagnasco, A. y Trigilia, C. (1984): Società e politica nelle aree di piccola impresa. Il caso Bassano, Venecia, Arsenale.

- (1985): Societd e politica nelle aree di piccola impresa. Il caso della Valdelsa, Milán, Angeli.

Becattini, G. (1987): Mercato e forze locali: il distretto industriale, Bolonia, Il Mulino.

Bianchi, F. (1988): Sindacato e comunicazione politica. Una crisi non superata, Tesis de Doctorado en Sociología de1 trabajo, Facultad de Ciencias Políticas de Florencia.

Brunetta, R. y Dal Co, M. (1987): Rapporto '87. Lavoro e politiche dell'occupazione in Italia, Roma, Ministerio del Trabajo y de la Previsión Social.

Caciagli, M. (1987): «L'iscrizione dei giovani ai partiti in Europa», en Politica y Sociedad, Madrid, CIS-CESCO, vol. I.

Caciagli, M. y Scaramozzino, P. (1983): Il voto di chi non vota, Milán, Comunità. Capecchi, V. y Pesce, A. (1983): «Se Ia diversitá è un valore», in Inchiesta, número. 59-60.

Carrieri, M. y Donolo, C. (1989): L'incerta rappresentanza, sintesis de la ponencia presentada en los Encuentros: Dopo $i$ Cobas: questioni sulla rappresentativitd̀ sindacale, Siena, 20-21 enero. 
El sindicato en Italia: ¿una crisis de reptesentacion?

Cella, G.P. (1981): «Tra rappresentatività e rappresentanza», $I l$ progetto, número 1 .

- (1981): «Tra interessi e solidarietà: l'azione sindacale nella crisi del pluralismo», Stato e mercato, núm. 2.

Cella, G. P, y Treu, T. (1988): Relazioni industriali, Bolonia, Il Mulino.

Cesos (1988): Le relazioni sindacali in Italia; rapporto 1986-1987, Roma, Ed. Lavoro.

David, P. y Vicarelli, G. (1983): L'azienda famiglia, Bari, Laterza.

Donolo, C. (1981): «Il sindacato nella crisi della rappresentanza», Quaderni di rassegna sindacale, núm. 90/91.

Ferraresi, F. (1981): Il sindacato nella pubblica amministrazione, Roma, E. Lavoro.

Gallino, L. (1985) (a cargo de): Il lavoro e il sto doppio, Bolonia, Il Mulino. Giovannini, P. (1982): I giovani e la crisi del lavoro, Flotencia, Op. Univ.

- (1985): «Sindacato e questione giovanile», Il ponte, núm. 2.

- (1985): «Dalla Grande Impresa al Piccolo Socialismo», Il ponte, núms. 5-6.

- (1988): «Generazioni e mutamento politico in Italia», Rivista italiana di Scienza Politica, núm. 3.

Inglehart, R. (1977): The Silent Revolution in Europe, Princeton, N. Y., Princeton University Press.

ISTAT-AIS (1988): Immagini della società italiona, Roma, ISTAT.

Manghi, B. (1981): Democrazia minima, Roma. Ed. Lavoro.

- (1987): Passaggio senxa riti, Roma, Ed. Lavoro.

Melucci, A. (1986): Movimenti sociali e sistema politico, Milán, Angeli.

Offe, C. (1977): Lo stato nel capitalismo maturo, Milán, Etas libri.

Paci, M. (1973): Mercato del lavoro e classi sociali in Italia, Bolonia, Il Mulino. -. (1982): La struttura sociale italiana, Bolonia, Il Mulino.

Parisi, A. y Pasquino, G. (1977): Relazioni partiti-elettori e tipo di voto, en Parisi-Pasquino, Continuita e mutamento elettorale in Italia, Bolonia, Il Mulino.

Pasquino, G. (1983): «Rapporti di conflitto e rapporti di collabotazione», Quaderni di rassegna sindacale, núm. 104.

Piore, M. y Sabel, C. (1985): Keynesismo internazionale e specializzazione flessibile, en Carrieri, M. y Perulli, P., Il teorema sindacale, Bolonia, Il Mulino.

Pizzorno, A. (1980): I soggetti del pluralismo. Classi, Partiti, Sindacati, Bolonia, Il Mulino.

Regalia, I. (1981): «Sindacalizzazione e mutamento del mercato e del lavoro», Quaderni di rassegna sindacale, núms. 90-91.

- (1986): Democrazia e sindacato, en Il futuro del sindacato, a cargo de Ferrante, G., Roma, Ediesse.

Regini, M. (1988): La sfida della flessibilitd, Milano, Angeli.

Regini, M. y Regalia, I. (1982): «Sindacato, Instituzioni, Sistema Politico» en Relazioni industriali, a cargo de Cella, G. P. y Treu, T., Bolonia, It Mulino. Romagnoli, G. (1980): La sindacalizzazione tra ideologia e pratica, Roma, E. Lavoro. 
«Papers»: Revista de Sociologia

Romagnoli, G. (1981): «Ripensare la rappresentanza per riformare l'organizzazione», Prospettive sindacali, núm. 1.

Sabel, C. y Zeitlin, J. (1982): «Alternative storiche alla produzione di massa», Stato e mercato, nútn. 5.

Salvati, M. (1984): Economia e politica in Italia dal dopoguerra ad oggi, Milán, Garzanti.

Sartori, G. (1987): «Rappresentanza», en Elementi di teoria politica, Bolonia, Il Mulino.

Schumpeter, Y. (1927): «Le classi sociali in ambiente etnicamente omogeneo», en Sociologia dell'imperialismo, Bari, Laterza, 1972.

Signori, C. (1988): La rappresentanza difficile. Una ricerca sui giovani e il sindacato, Tesis de Doctorado en Sociología del trabajo, Facultad de Ciencias Políticas de Florencia.

Surtenti, G. (1981): «Rappresentare il sommerso», $I l$ progetto, núm. 3.

Sylos Labini, P. (1986): Le classi sociali negli anni ottanta, Bari, Laterza.

Trigilia, C. (1985): «La regolazione localistica: economia e politica nelle aree di piccola impresa, en Stato e mercato, núm. 14.

- (1986): Grandi partiti e piccole imprese, Bolonia, Il Mulino.

Varaldo, R. (1979: Ristrutturazioni industriali e rapporti tra imprese, Milán, Angeli. 